\title{
Measurements of parametric instabilities at laser intensities relevant to strong shock generation
}

\author{
G. Cristoforetti, ${ }^{1}$ L. Antonelli, ${ }^{2}$ S. Atzeni, ${ }^{2}$ F. Baffigi, ${ }^{1}$ F. Barbato, ${ }^{3}$ D. Batani, ${ }^{4}$ G. Boutoux,${ }^{4}$ \\ A. Colaitis, ${ }^{4}$ J. Dostal, ${ }^{5,6}$ R. Dudzak, ${ }^{5,6}$ L. Juha, ${ }^{5,6}$ P. Koester, ${ }^{1}$ A. Marocchino, ${ }^{2,7}$ \\ D. Mancelli, ${ }^{4,8}$ Ph. Nicolai, ${ }^{4}$ O. Renner, ${ }^{6,5}$ J. J. Santos, ${ }^{4}$ A. Schiavi, ${ }^{2}$ M. M. Skoric, ${ }^{9}$ \\ M. Smid, ${ }^{6}$ P. Straka, ${ }^{6}$ and L. A. Gizzi ${ }^{1}$ \\ ${ }^{1}$ Intense Laser Irradiation Laboratory, INO-CNR, 56124 Pisa, Italy \\ ${ }^{2}$ Dipartimento SBAI, Università di Roma "La Sapienza," 00185 Roma, Italy \\ ${ }^{3}$ Empa Swiss Federal Laboratories for Materials Science and Technology, 8600 Dübendorf, Switzerland \\ ${ }^{4}$ Université Bordeaux, CNRS, CEA, CELIA, UMR 5107, F-33405 Talence, France \\ ${ }^{5}$ Institute of Plasma Physics, Czech Academy of Sciences, 18200 Prague 8, Czech Republic \\ ${ }^{6}$ Institute of Physics, Czech Academy of Sciences, 18221 Prague 8, Czech Republic \\ ${ }^{7}$ Laboratori Nazionali di Frascati, 00044 Frascati, Rome, Italy \\ ${ }^{8}$ Donostia International Physics Center (DIPC), 20018 Donostia/San Sebastian, Basque Country, Spain \\ ${ }^{9}$ National Institutes of Natural Sciences, 105-0001 Tokyo, Japan
}

(Received 21 September 2017; accepted 18 December 2017; published online 8 January 2018)

Parametric instabilities at laser intensities in the range $(2-6) \times 10^{15} \mathrm{~W} / \mathrm{cm}^{2}(438 \mathrm{~nm}, 250 \mathrm{ps}$, $100-300 \mathrm{~J}$ ) have been investigated in planar geometry at the Prague Asterix Laser System facility via calorimetry and spectroscopy. The density scalelength of the plasma was varied by using an auxiliary pulse to form a preplasma before the arrival of the main laser beam and by changing the delay between the two pulses. Experimental data show that Stimulated Brillouin Scattering (SBS) is more effective than Stimulated Raman Scattering (SRS) in degrading laser-plasma coupling, therefore reducing the energy available for the generation of the shock wave. The level of the SBS backscatter and laser reflection is found to be in the range between 3\% and $15 \%$ of the incident laser energy, while Backward SRS (BRS) reflectivity ranges between $0.02 \%$ and $0.2 \%$, depending on the delay between the pulses. Half-integer harmonic emission is observed and provides a signature of Two Plasmon Decay (TPD) occurring around the quarter of the critical density. Data analysis suggests that SRS is driven in beam speckles with high local intensity and occurs in bursts, particularly at higher laser intensities, due to the presence of kinetic mechanisms saturating the SRS growth in the speckles. Time-resolved measurements also show that BRS occurs in the trailing part of the laser pulse, when the plasma has a longer density scalelength. Our measurements also indicate that hot electrons are predominantly produced by SRS rather than TPD. Published by AIP Publishing. https://doi.org/10.1063/1.5006021

\section{INTRODUCTION}

Shock ignition (SI) is a promising two-step scheme of Inertial Confinement Fusion (ICF), where a strong converging shock wave is launched at the end of the compression phase to ignite the fuel. ${ }^{1-4}$ Both the compression of the DT pellet and the igniting shock wave can be produced by a single tailored laser pulse, consisting of a ns long peak at moderate intensity lower than $10^{15} \mathrm{~W} / \mathrm{cm}^{2}$ followed by a short intense spike $(300-500 \mathrm{ps})$ at intensity between $10^{15}$ and $10^{16} \mathrm{~W} / \mathrm{cm}^{2}$. The SI approach may allow ignition with a low-velocity implosion, reducing the growth of the Rayleigh-Taylor Instability. In addition, SI is robust with respect to non-uniform spike irradiation and shock synchronization, as predicted by hydrodynamic simulations ${ }^{5,6}$ and leads to high gain, ${ }^{7}$ possibly enabling ignition at moderate laser energies, which are already available at Laser Megajoule $(\mathrm{LMJ})^{8}$ and National Ignition Facility (NIF) facilities. ${ }^{9}$ In this context, an extensive preparatory study has been carried out also in the framework of the HiPER project ${ }^{10}$ and is now aiming at full scale demonstration at one of the above facilities.
The success of the SI concept depends mainly on the coupling of the laser spike with the extended corona surrounding the imploding shell, where an efficient laser absorption, which is able to generate a strong shock wave ( $>300 \mathrm{Mbar}$ ), is needed. In recent experiments carried out at the OMEGA laser $^{11,12}$ in spherical irradiation geometry, at laser intensities relevant for SI $\left(\mathrm{I} \sim 6 \times 10^{15} \mathrm{~W} / \mathrm{cm}^{2}\right)$, a peak ablation pressure close to $400 \mathrm{Mbar}$ was inferred, which constitutes a significant breakthrough towards the demonstration of the feasibility of the SI scheme. Despite this step forward, the physics of the laser-plasma interaction in this highly non-linear regime is still largely unknown and needs dedicated investigations. In particular, the growth of parametric instabilities such as Stimulated Brillouin Scattering (SBS), Stimulated Raman Scattering (SRS), and Two-Plasmon Decay (TPD), and their interplay, can be considerable, and laser filamentation can further enhance their role in the interaction. These processes can significantly degrade laser-plasma coupling due to a strong reflection of light (SBS and SRS), resulting in the absorption of laser energy in rarefied regions of the corona, far from the ablation layer. Moreover, TPD and SRS generate electron plasma waves that lead to suprathermal electrons via damping. 
Such electrons, depending on their energy,,$^{2,11-15}$ may preheat the fuel or affect the shock pressure.

In the last few decades, parametric instabilities have been deeply investigated in conditions suitable for directdrive and indirect-drive schemes ${ }^{16}$ of ICF. The majority of these studies, therefore, refers to an interaction regime significantly different from that envisaged for the SI scheme, with laser intensities a factor 10 lower, leaving the interaction regime of interest for SI almost unexplored. The extrapolation of these studies to SI conditions is made complex by the high nonlinearity of parametric instabilities in this regime. After a rapid boost, instabilities can show a saturation due to non-linear effects limiting the growth of electron plasma waves (EPWs) or ionic acoustic waves (IAWs) in the interaction region. EPW decay via Langmuir Decay Instability ${ }^{17,18}$ and ponderomotive trapping of thermal electrons in the EPW field are classical examples of phenomena, leading to EPW saturation. More recently, other nonlinear mechanisms, such as bowing and filamentation of EPW into laser speckles, ${ }^{19,20}$ collective speckle effects, ${ }^{21}$ or instability cascades, have been suggested. All these mechanisms produce a phase detuning of the waves, which in many cases results in consecutive stages of damping and excitation of instabilities, in a burst-like, chaotic behavior. ${ }^{22,23}$ A fully kinetic approach is therefore needed to model processes as non-local heat transport or Landau damping in the kinetic regime..$^{19,24,25}$

Recently, several attempts have been made to model laser-plasma interactions in SI conditions with fully kinetic massively parallel PIC codes. According to Riconda et al. ${ }^{26}$ and Klimo et al., ${ }^{27,28}$ most of the laser energy is absorbed at densities lower than $n_{c} / 4$, where $n_{c}$ is the critical density for the main beam, and the reflectivity due to parametric instabilities, temporally bunched in trains of bursts, can span from the percent level to $30 \%-50 \%$ of the laser energy. Simulations show the importance of kinetic effects, leading to ps- or subps-long bursts of SRS and SBS, where SRS sometimes occurs in the inflationary regime, and to nonMaxwellian distributions of electron energies. Simulations also reveal the importance of modelling 2D and 3D effects such as filamentation, laser spraying, cavitation, or sidescattered EPW/light. Unfortunately, 2D numerical simulations carried out in the density range $0.01-0.3 n_{c}$ are at present limited to a few picoseconds of the interaction, which is much shorter than the ignition spike and which is inadequate to model processes having a small growth rate, such as filamentation. While particle in cell simulations (of the VlasovFokker-Planck equation) are limited to a few picoseconds due to their computation costs, a series of reduced-hydrodynamic models are being investigated to partially take into account some effects that might influence the nanosecond plasma evolution, such as the nonlocal electron transport, ${ }^{29}$ the growth of parametric instability, the generation of fast electrons, ${ }^{30}$ and the influence of self-induced magnetic fields. ${ }^{31}$

A few experiments carried out at OMEGA ${ }^{11,12,32,33}$ and LULI facilities ${ }^{34-36}$ investigated the laser plasma interaction at SI intensities. The overall energy scattered by SRS/SBS in these experiments is disparate, in a range going from a few percent up to $\sim 40 \%-50 \%$ of the incident energy, strongly dependent on the irradiation geometry and on the laser intensity. SBS back-reflectivity is found to be around $10 \%$ in all the experiments, rising to $\sim 20 \%$ when side-scattered light is also considered. ${ }^{35} \mathrm{SRS}$ reflectivity shows a larger range of variability, going from a few percent in planar irradiation experiments $^{33-35}$ up to $\sim 36 \%$ in spherical geometry. ${ }^{11,32}$ SI experiments at the OMEGA laser also suggest that SRS is the dominant mechanism of generation of hot electrons (HE), showing a conversion efficiency as high as $\sim 9 \%$ of the laser energy in the case of spherical irradiation. Furthermore, they suggest that HE can significantly contribute to the shock formation by increasing the ablation pressure by $\sim 30 \%{ }^{9}$ Both the variability of SRS and its effects on the fuel compression call for additional investigation. In particular, the dependence of SRS threshold and saturation on the beam speckle size needs further attention.

In this paper, we report and discuss experimental data obtained in a series of experiments carried out in a planar geometry at the Prague Asterix Laser System (PALS) ${ }^{37}$ at intensities of interest for the Shock Ignition interaction regime. The overall description of the diagnostics used and an overview of the experimental results obtained, including the measured pressure of the shock wave, are reported elsewhere. ${ }^{38-42}$ Here, we focus on the laser plasma interaction and in particular, on the Stimulated Raman Scattering and Two Plasmon Decay instabilities. Despite our density scalelength $(\sim 100 \mu \mathrm{m})$ and the electron temperature $(\sim 1.5-2 \mathrm{keV})$ being lower than those envisaged in a real SI scenario, the data reported here provide a comprehensive study of the growth of parametric instabilities at a laser intensity relevant for SI where very little experimental data exist and where, as discussed above, a strong nonlinearity and interplay between different processes are expected to play a dominant role.

\section{EXPERIMENTAL SETUP}

A basic scheme of the experimental setup relevant to the results discussed here is shown in Fig. 1, while the complete setup is described elsewhere. ${ }^{38}$

The interaction beam, frequency converted into the $3 \mathrm{rd}$ harmonic $\left(\lambda_{0}=438 \mathrm{~nm}\right)$ of the fundamental laser frequency and with a duration of 250 ps (FWHM), was smoothed by means of a Random Phase Plate (RPP) and focused on the target at normal incidence by an $\mathrm{f} / 2$ optical system. The beam profile in the focal spot and the effective energy enclosed in it were accurately measured by imaging and calorimetric techniques. RPP resulted in a Gaussian beam profile of $\sim 100 \mu \mathrm{m}$ (FWHM) and peak intensity in the range between 2 and $6 \times 10^{15} \mathrm{~W} / \mathrm{cm}^{2}$, depending on the energy of the laser shot.

In some shots, an auxiliary beam (not shown in Fig. 1) at the fundamental wavelength $\left(\lambda_{0}=1314 \mathrm{~nm}\right.$ and $\mathrm{FWHM} \approx 250 \mathrm{ps}$ ) and incident at $30^{\circ}$ with respect to the target normal impinged on the target before the main pulse and generated an extended preplasma, mimicking the corona at the end of the compression phase in the shock ignition scheme. The auxiliary beam had a focal spot diameter of $\sim 900 \mu \mathrm{m}$ (FWHM), i.e., much larger than the focal spot of the main beam, to reduce $2 \mathrm{D}$ effects during the interaction, resulting in a laser intensity of $\sim 7 \times 10^{13} \mathrm{~W} / \mathrm{cm}^{2}$. The delay 


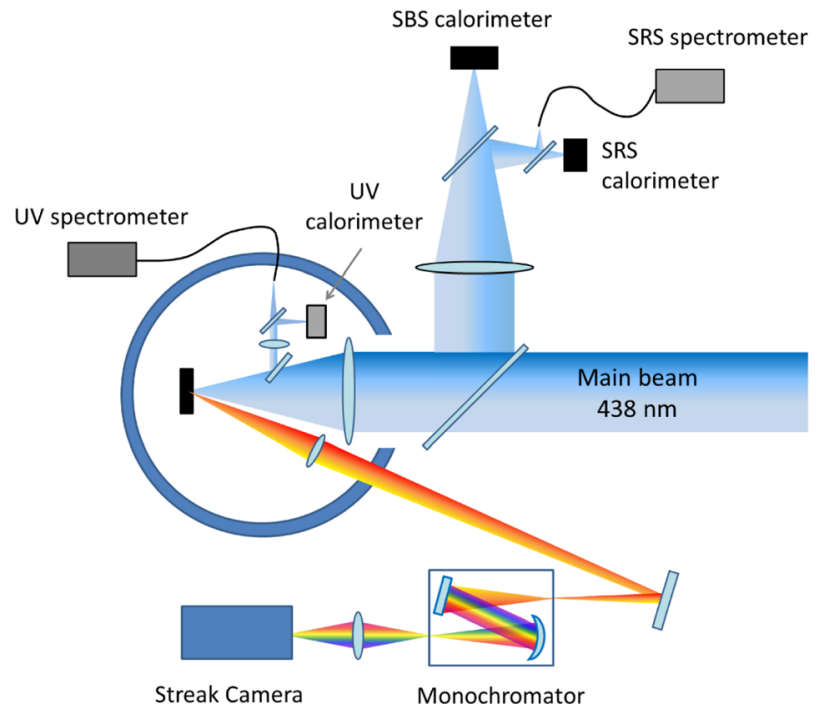

FIG. 1. Experimental setup used for the investigation of parametric instabilities.

between the peaks of auxiliary and main pulses was varied in the range between 0 and 1200 ps to change the density scalelength of the preplasma.

Thin multilayer targets consisting of a layer of plastic $\left(\mathrm{C}_{8} \mathrm{H}_{7} \mathrm{Cl}\right.$, parylene-C) with thicknesses ranging from 10 to $180 \mu \mathrm{m}$ on the irradiated side, one or two tracer layers ( 5 to $10 \mu \mathrm{m})$ of $\mathrm{Cu}$ and $\mathrm{Ti}$ and in some shots a $25 \mu \mathrm{m}$-thick $\mathrm{Al}$ layer on the rear side, were used. The low- $\mathrm{Z}$ material on the front played the role of the ICF ablator material, while chlorine ions allowed the plasma temperature to be measured via high-resolution X-ray spectroscopy. Both the spectra and the energy of the light backscattered during the laser plasma interaction were found not to depend on the thickness of the plastic layer nor on the presence of the $\mathrm{Al}$ layer. Therefore, these features of the targets are here disregarded. The layers of $\mathrm{Cu}$ and $\mathrm{Ti}$ were used as markers of hot electrons via $\mathrm{K}_{\alpha}$ photon emission, which was detected using a CCD operating in the single-photon regime ${ }^{43}$ and using two spherically bent quartz (422) and (203) crystal imaging spectrometers. Both $\mathrm{K}_{\alpha}$ and X-ray spectrometers used Kodak AA400 films as detectors. A Bremsstrahlung spectrometer using $k$-edge and differential filtering (14 filters of increasing $\mathrm{Z}$ from $\mathrm{Al}$ to $\mathrm{Pb}$ ) was also used with Imaging Plates to measure the $x$-ray fluence and, indirectly, infer a slope temperature for the hot electron distribution. ${ }^{44}$ This spectrometer was looking at the front side of the target at $\sim 30^{\circ}$ from the laser axis.

The backscattered radiation originating from SRS and SBS/laser reflection was collected by the focusing lens and measured using two calorimeters. On the same line, the radiation was spectrally dispersed by a compact, fiber Vis-IR spectrometer $\left(\Delta \lambda_{\text {res }} \approx 1 \mathrm{~nm}\right)$, enabling the investigation of backscattered light up to $\omega_{0} / 2(\lambda \sim 876 \mathrm{~nm})$, originating from SRS at densities lower than $n_{c} / 4$ and SRS/TPD instabilities occurring near $n_{c} / 4$. Such spectral resolution, however, did not allow the contribution of SBS to be separated from that of laser reflection in the backscattered emission at $\lambda \sim 438 \mathrm{~nm}$. Light at $3 / 2 \omega_{0}$ harmonics of laser frequency $(\lambda \sim 292 \mathrm{~nm})$, originating from TPD, was collected inside the vacuum chamber and sent to a UV spectrometer $\left(\Delta \lambda_{\text {res }} \approx 0.3 \mathrm{~nm}\right)$ and a calorimeter. Raman scattered light was also collected at a slightly larger angle than the focusing cone $\left(\approx 20^{\circ}\right)$, spectrally dispersed by a monochromator, and relayed onto the entrance slit of a Hamamatsu C7700 Streak Camera. A spectral range of $100 \mathrm{~nm}$ was detected on the camera at a maximum temporal resolution of $8 \mathrm{ps}$.

\section{INTERACTION CONDITIONS}

The interaction conditions of the main pulse, with and without the auxiliary pulse, were modelled via hydrodynamic simulations performed with the codes DUED ${ }^{45}$ and CHIC. ${ }^{46}$ Figure 2 shows the instantaneous values of temperature and density scalelength $L=n_{e} /\left(d n_{e} / d x\right)$ at densities $n_{c} / 4$ and $0.12 n_{c}$ for a laser intensity of $I_{\max }=2.9 \times 10^{15} \mathrm{~W} / \mathrm{cm}^{2}$ and a delay of 600 ps between auxiliary and main pulses. The $n_{c} / 4$ and $0.12 n_{c}$ densities are the plasma regions where TPD and Backward SRS (BRS) are driven in the present experiment.

Figure 2(a) shows that the density scalelength rapidly increases during the pulse. The values of the scalelength at $n_{\mathrm{c}} / 4$ and $n_{\mathrm{c}} / 12$ are in the range between 20 and $80 \mu \mathrm{m}$ and
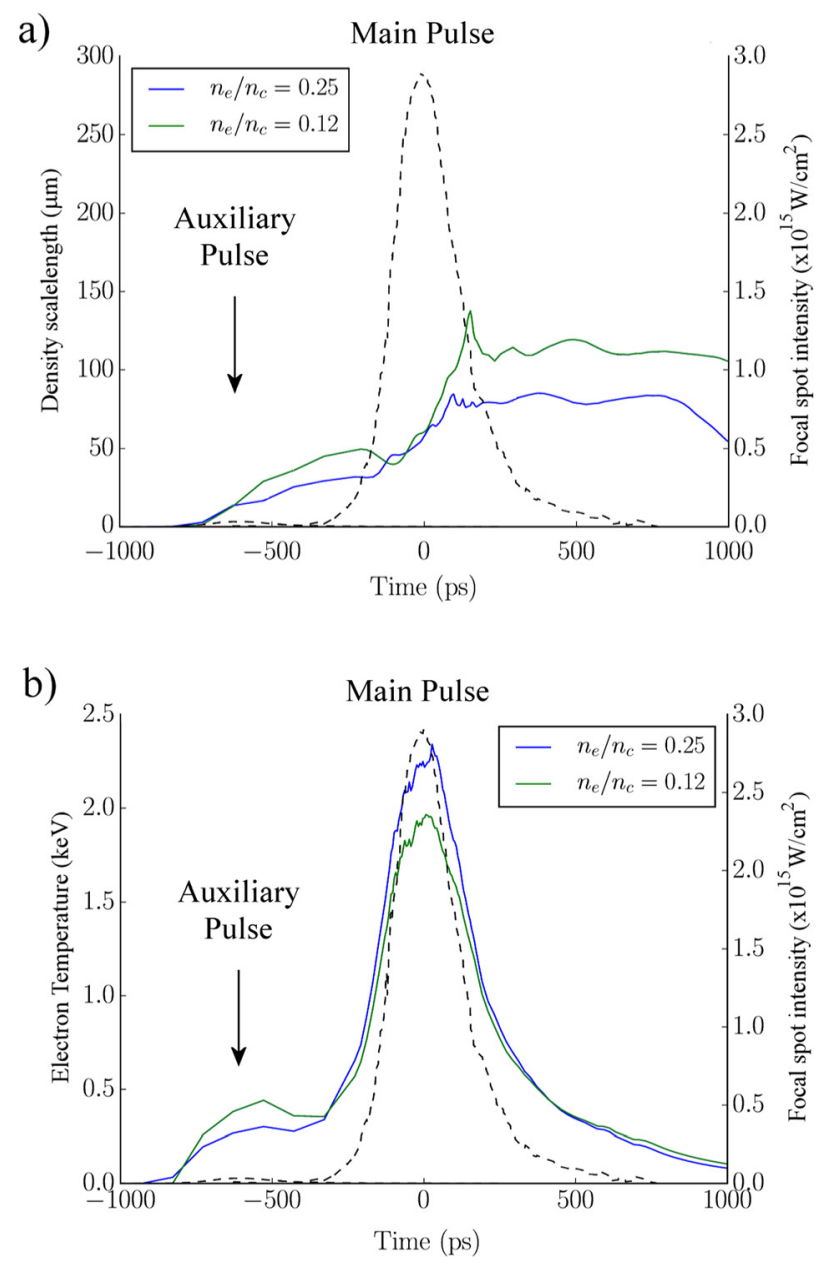

FIG. 2. Instantaneous values of the density scalelength (a) and temperature (b) computed at $n_{c} / 4$ and $0.12 n_{c}$ by the CHIC code for a laser intensity $I_{\max }$ $=2.9 \times 10^{15} \mathrm{~W} / \mathrm{cm}^{2}$ and a delay of $600 \mathrm{ps}$ with respect to the auxiliary pulse. The intensity of the laser pulse is indicated as a gray dashed line and corresponds to the right-hand side ordinate axis. 
between 40 and $150 \mu \mathrm{m}$, respectively, depending on the time and on the delay between the pulses. The scalelength increases with the delay between main and auxiliary pulses, showing a saturation at the longest delays explored. The preplasma scalelength given by the simulations is also in agreement with the 2D plasma density profiles measured by interferometric techniques (monitor online) ${ }^{38}$ and X-ray laser (XRL) deflectometry. ${ }^{39}$

According to Fig. 2(b), simulations show a timedependent temperature, reaching maximum values between $1.9 \mathrm{keV}$ and $2.5 \mathrm{keV}$, slightly depending on the laser intensity and density region, in the range of densities $0.10-0.25 n_{c}$. According to the simulations, the temperature of the plasma during the main peak irradiation does not significantly depend on the delay between the pulses, with variations lower than $10 \%$, but is determined by the energy of the main pulse. Spatially resolved X-ray spectra (not displayed here), showing well resolved $\mathrm{He}$ - and Li-like lines from $\mathrm{Cl}$ ions, were compared with SPECT3D predictions, ${ }^{47}$ providing a temperature averaged over time of $\approx 700-850 \mathrm{eV}$ for all shots. Such values, however, refer to a plasma overdense region, for which hydrodynamic simulations predict temperatures of $\approx 800-1000 \mathrm{eV}$ at the time of the main laser peak. The time-averaged value of the temperature of the preplasma produced by the auxiliary pulse was also inferred by $\mathrm{Cl} \mathrm{X}$ ray spectroscopy. In this case, a value of $\sim 175 \mathrm{eV}$ was obtained for the underdense region, which is lower than the peak value of $\sim 300-400 \mathrm{eV}$ obtained by simulations.

\section{CALORIMETRY}

Here, we present the results of backscattered energy, namely, calorimetric measurements of the energy backscattered into the cone of the focusing lens. As already presented in Koester et al., ${ }^{38}$ backscattering is dominated by light around $438 \mathrm{~nm}$, due to SBS and laser reflection, and ranges between 3\% and 15\% of the incident laser energy [Fig. 3(a)]. On the other hand, SRS gives rise to backscattered light in the $630-750 \mathrm{~nm}$ spectral range, consisting of $\sim 0.02 \%-0.2 \%$ of laser energy [Fig. 3(b)]. Both these values show a clear increasing trend with the auxiliary-main pulse delay, where SBS and SRS reflectivities grow by factors 2 and 5, respectively, when the delay increases from 0 to $1200 \mathrm{ps}$. A similar trend is found for the intensity of scattered light in the spectral range $600-800 \mathrm{~nm}$ due to SRS [Fig. 3(b)]. This behavior can be explained by considering the inhomogeneity of the plasma which determines the convective gain of these instabilities. Regarding the trend in Fig. 3(a), hydrodynamic simulations show that the amount of backscattered laser light does not increase with the delay between the pulses; therefore, we expect that the trend observed is mainly determined by the growth of SBS instability with the delay. In our interaction conditions, however, the SBS threshold and the convective gain are mainly ruled by the gradient of the expansion velocity rather than by the density scalelength. ${ }^{48}$ By using the approximate SBS threshold taken from Kruer $^{49}$ and the profiles obtained by hydrosimulations for shots without the auxiliary pulse, we find that the SBS threshold in the underdense plasma, which is determined by a)
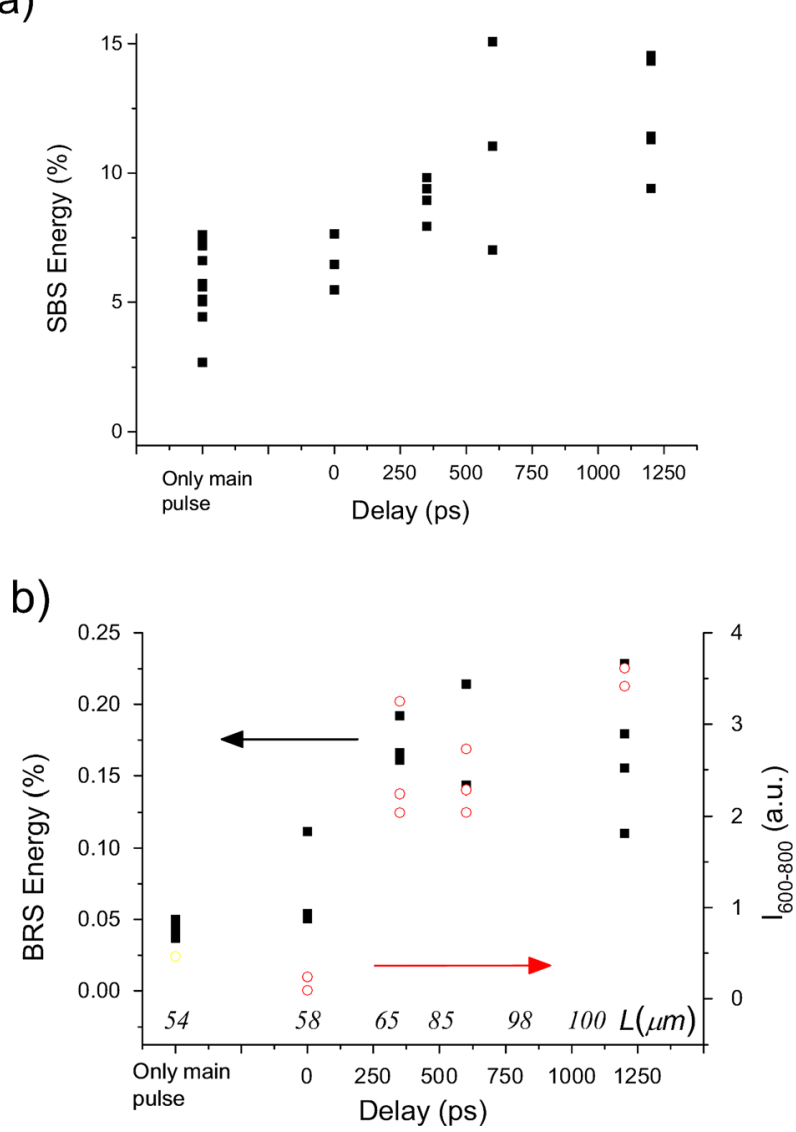

FIG. 3. (a) Energy backscattered by SBS and laser backscatter $(\lambda=438 \mathrm{~nm})$. (b) SRS energy (black squares) and spectral intensity $\mathrm{I}_{600-800}$ integrated in the range $600-800 \mathrm{~nm}$ (red circles) versus the delay auxiliary-main pulse for $I_{\max } \approx(3.5-6) \times 10^{15} \mathrm{~W} / \mathrm{cm}^{2}$. The values of the density scalelength calculated by the code DUED in proximity of the laser peak $\left(I_{\text {max }} \approx 5 \times 10^{15}\right.$ $\mathrm{W} / \mathrm{cm}^{2}$ ) and at a density $n_{e}=0.12 n_{c}$ are also reported for fixed values of auxiliary-main pulse delay.

the velocity gradients, is $I_{\text {thres }} \sim(1-2) \times 10^{15} \mathrm{~W} / \mathrm{cm}^{2}$, while the threshold calculated by considering the density gradient is an order of magnitude lower. Due to the poor spectral resolution, however, we were unable to determine accurately the plasma density region where SBS is driven. Hydrodynamic simulations including the auxiliary pulse clearly show that the gradient of the expansion velocity is strongly reduced in the underdense plasma, therefore lowering the SBS threshold and correspondingly boosting the instability gain. This can possibly explain the experimental results plotted in Fig. 3(a).

Figure 3(b), on the other hand, can be easily explained by considering that larger delays produce longer plasmas, resulting in larger gain for the SRS, whose threshold is strongly affected by the density scalelength in the plasma. In order to clarify the relationship between the auxiliary-main pulse delay and the density gradient, the values of the density scalelength calculated by the code DUED in the proximity of the laser peak $\left(I_{\max } \approx 5 \times 10^{15} \mathrm{~W} / \mathrm{cm}^{2}\right)$ and at a density $n_{e}=0.12 n_{c}$ are reported in Fig. 3(b) for fixed values of the delay.

Calorimetric measurements of $3 / 2 \omega_{0}$ and $\omega_{0} / 2$ emission, obtained by using an approximate isotropic distribution, 
give a conversion efficiency $\left(\eta_{3 / 2}\right)$ of around $0.5 \%$ of incident energy and a corresponding value $\eta_{1 / 2}$ in the range $(0.4-2) \times 10^{-2} \%$, i.e., a negligible loss of laser energy. Since the actual angular dependence is not known, such values have to be considered only as an order of magnitude.

\section{HALF-INTEGER HARMONICS OF LASER FREQUENCY}

Typical $\omega_{0} / 2(\lambda=876 \mathrm{~nm})$ and $3 / 2 \omega_{0}(\lambda=292 \mathrm{~nm})$ spectra are shown in Fig. 4. Both spectra exhibit an energy splitting around the harmonic wavelength, which is related to the frequency difference of plasma waves produced by TPD instability at $n_{e} \approx n_{c} / 4$.

The frequency shift of TPD blue and red EPWs from the central frequency $\omega_{0} / 2$ can be expressed by

$$
|\delta \omega| / \omega_{0}=\frac{9}{4}\left(v_{t h}^{2} / c^{2}\right) \kappa
$$

where $\kappa=\mathbf{k}_{\mathbf{B}} \cdot \mathbf{k}_{\mathbf{0}} / k_{0}^{2}-1 / 2, k_{B}$ is the blue EPW wavevector, and $v_{t h}$ is the thermal velocity. ${ }^{50}$
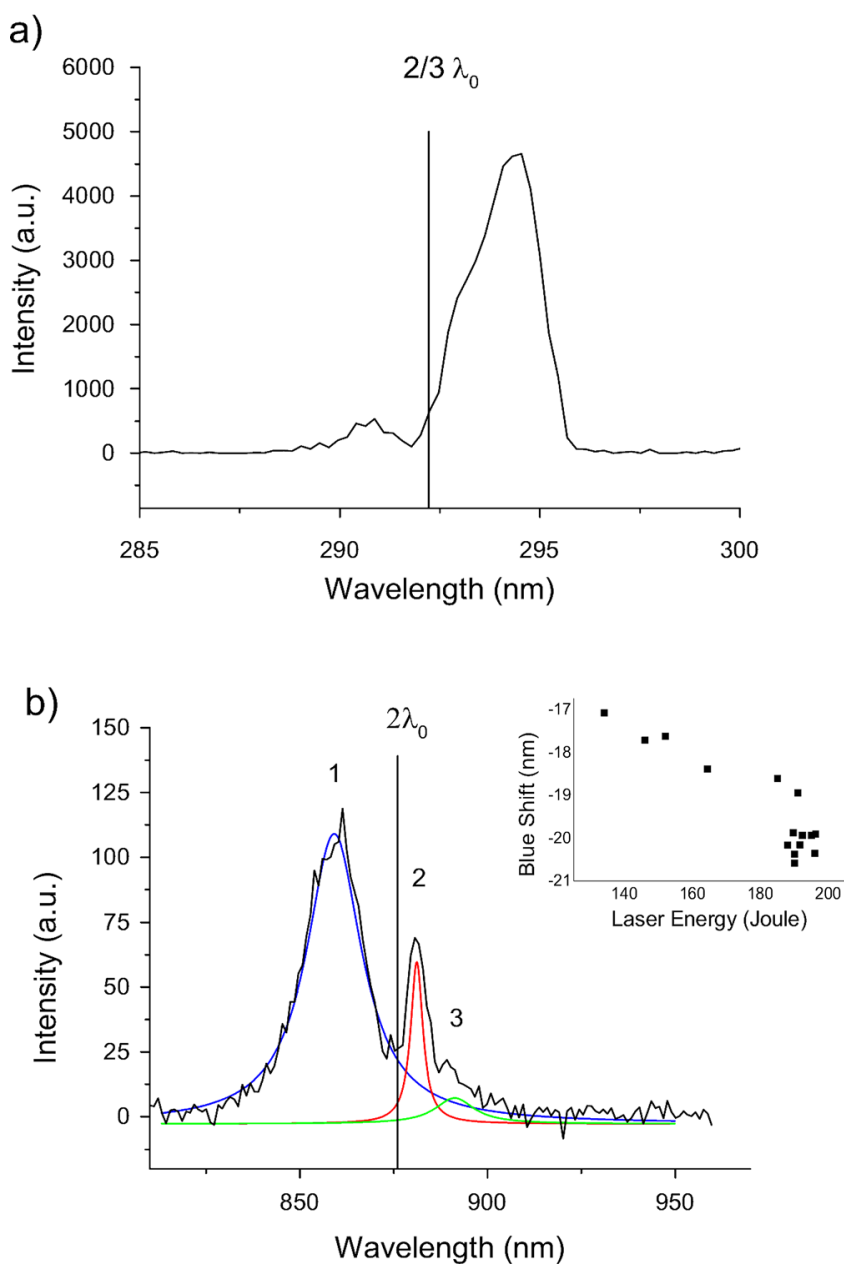

FIG. 4. Typical $3 / 2 \omega_{0}$ and $\omega_{0} / 2$ spectra, obtained at $I_{\max } \approx 5 \times 10^{15} \mathrm{~W} / \mathrm{cm}^{2}$ (black lines). Vertical lines indicate the position of the nominal laser harmonics. Red, green, and blue lines in (b) show the peaks resulting by fitting the spectrum using 3 Lorentzian peaks. The inset in (b) shows a plot of the shift of the blue peak 1 vs. the energy of the laser pulse. Adapted with permission from Cristoforetti et al., Europhys. Lett. 117, 35001 (2017), Copyright 2017 EPL Association. ${ }^{65}$
The well-defined structure of $\omega_{0} / 2$ [Fig. 4(b)] agrees with the relevant literature on the topic. ${ }^{51,52}$ Besides the evident blue- and red-shifted peaks (labelled 1 and 2, respectively), a less evident bump at longer wavelengths (peak 3) is visible, which we identify as a supplementary peak, as in Seka et al..$^{51}$ The blue peak has a shift from the nominal $2 \lambda_{0}$ wavelength and a width significantly larger than the red peak 2, approximately 3 and 3.6 times, respectively. Conversely, the shift of peak 1 is similar to the shift of the weak peak 3 . The different frequency shift of peaks 1 and $3\left(\overline{\Delta \omega_{1,3}} / \omega_{0}\right.$ $\left.=1.1 \times 10^{-2}\right)$ and peak $2\left(\overline{\Delta \omega_{2}} / \omega_{0}=3.4 \times 10^{-3}\right)$ indicates that the EPWs responsible for them have different wavevectors and a different origin. This is possible since TPD instability in an inhomogeneous plasma can generate different EPWs in the range allowed by the Landau damping.

Both frequency shifts increase with laser energy, as visible in the inset of Fig. 4(b) for the blue peak. In previous works ${ }^{51}$ the sharp peak 2 was observed at laser intensities higher than TPD threshold but much lower than SRS threshold. For this reason, this is usually associated with a hybrid TPD/SRS instability rather than with a pure absolute SRS instability, where a pump electromagnetic wave decays in a forward electrostatic wave with $k \approx k_{0}$ (as TPD and SRS) and in a backward partly electrostatic and partly electromagnetic wave ${ }^{53}$ in the proximity of the $n_{c} / 4$ surface (here, $\left.n_{e} / n_{c} \approx 0.24\right)$. According to Seka et al. ${ }^{51}$ the shift of this peak in the $\omega_{0} / 2$ spectrum, differently from $3 / 2 \omega_{0}$ splitting, is well applicable for the estimation of the plasma temperature because it is not affected by the angle of observation or by geometrical effects, such as filamentation, cavitation, or 2D profiles. By using Eq. (1) with $\kappa=1 / 2$, we obtain a plasma temperature which increases from $1.35 \mathrm{keV}$ to $1.68 \mathrm{keV}$ when $I_{\max }$ rises from $2.4 \times 10^{15}$ to $3.7 \times 10^{15} \mathrm{~W} /$ $\mathrm{cm}^{2}$. This value agrees with plasma temperature given by simulations, considering that it is calculated from timeintegrated measurements; moreover, as shown below, it agrees with the Landau cutoff of the Raman spectrum produced at densities $n_{e}<n_{c} / 4$.

According to the literature, ${ }^{52}$ the broad peak 1 and the small peak 3 can be generated by Inverse Resonance Absorption (IRA) or by Raman Downscattering (RD) of a laser photon. In the IRA process, an EPW with $k_{\perp} / k_{0}<0.1$ is converted into a photon near its turning point; therefore, blue and red peaks are originated by conversion of blue and red EPWs, respectively. In the RD process, conversely, a laser photon is down-scattered by an EPW produced by TPD, so that blue and red peaks are generated by scattering with red and blue EPWs, respectively. Both IRA and RD need particular matching conditions, ${ }^{51,54}$ and many authors have speculated on the prevalence of the one or the other in different experiments. Here, both coupling conditions can be fulfilled near $n_{c} / 4$, where filamentation, turbulence, cavitation, and laser photon scattering can occur. For these reasons, it is here not possible to definitively exclude neither IRA nor RD. We note however that a blue shift $\Delta \lambda_{B} \approx 20 \mathrm{~nm}$ implies $\kappa \approx 1.7$, indicating the involvement of EPWs with wavevector $k_{e} \approx 2.3 k_{0}$. By taking a plasma temperature $T_{e} \approx 1.5 \mathrm{keV}$, as estimated above, we obtain $k_{e} \lambda_{D} \approx 0.27$, 

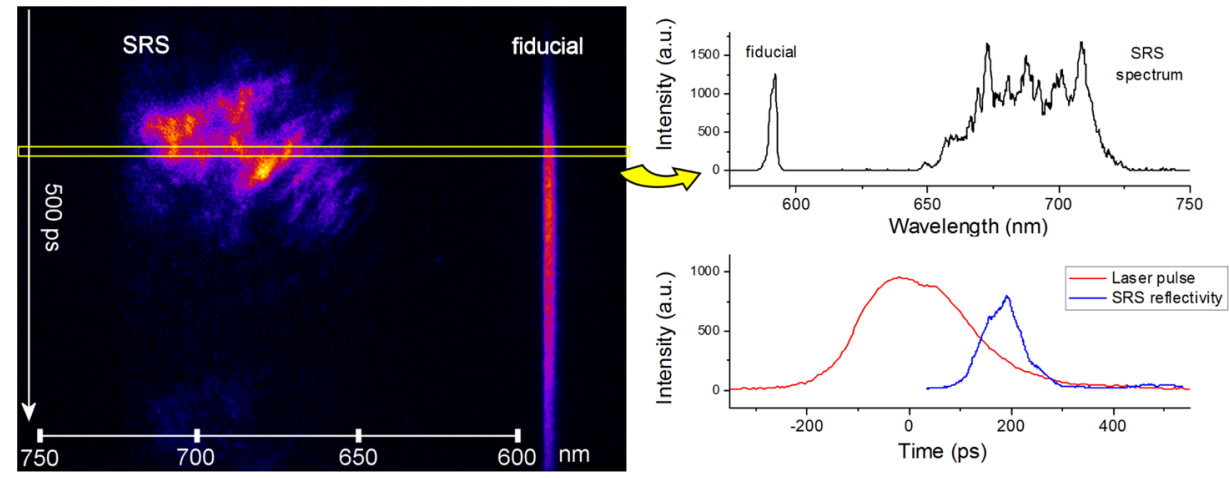

FIG. 5. (left) Time-resolved SRS spectrum obtained at a laser intensity of $I_{\max } \approx 3.4 \times 10^{15} \mathrm{~W} / \mathrm{cm}^{2}$ and no auxiliary pulse. The acquisition time window, spanning the vertical axis, is 500 ps. (top-right) SRS spectrum emitted in the selected time window of $\Delta \mathrm{t}=20$ ps, as shown on the left. (bottom-right) Temporal profiles of the laser pulse and SRS emission.

indicating that EPWs originating from the broad peaks 1 and 3 are located near the Landau cutoff at densities $n_{e} / n_{c} \approx 0.21-0.22$. According to the linear theory, ${ }^{55} \mathrm{TPD}$ driven in such a low density region is expected to occur in a convective regime, as previously found both in experiments and numerical calculations. ${ }^{51,56,57}$ Such modes could be generated by ion density fluctuations driven by the ponderomotive force of the EPWs driven at $n_{c} / 4$, which successively propagate down to lower densities. ${ }^{58}$ By using 2D PIC simulations with the OSIRIS code, Yan et al. showed that after a linear growth stage, such modes could be coupled with the SRS/TPD mode near the $n_{c} / 4$ surface via plasma waves and pump-depletion, giving rise to an intermittent burst-like pattern of the instability. ${ }^{22}$

Three-halve harmonic peaks are due to the coupling of laser photons with TPD EPWs. The blue peak is much weaker than the red one, which can be explained by the fact that the blue EPW must be reflected at its critical density in order that the $3 / 2 \omega_{0}$ blue peak is observed in the backscattering direction. ${ }^{50}$ Otherwise, the blue peak can be generated by the EPW resulting from the Langmuir Decay Instability of the primary TPD wave, as shown by Russel and DuBois. ${ }^{59}$ The frequency shift of blue and red peaks is $\overline{\Delta \omega_{3 / 2}} / \omega_{0}$ $\approx 1.09 \times 10^{-2}$, i.e., similar to that of peaks 1 and 3 of the $\omega_{0} / 2$ spectrum, suggesting that EPWs responsible for these harmonics are the same, located near the Landau cutoff.

The splitting of half harmonic spectra shows that TPD (including hybrid TPD/SRS instability) dominates on absolute SRS at the quarter critical density. This is in a qualitative agreement with 2D IC simulations by Weber and Riconda,${ }^{60}$ performed with values of temperature and density scalelength similar to our experiment. Simulations referring to hotter plasmas in SI conditions ${ }^{27,28}$ indicate instead a large contribution of absolute SRS at $n_{c} / 4$.

\section{BACKWARD STIMULATED RAMAN SCATTERING}

The SRS instability driven at densities lower than $n_{c} / 4$ gives rise to light scattered in the spectral range $\omega_{0} / 2$ $<\omega<\omega_{0}$. All the emission spectra measured in our experiment exhibit peaks in the $630-750 \mathrm{~nm}$ spectral range, as shown in the time resolved spectrum in Fig. 5, obtained with a laser intensity of $I_{\max } \approx 3.4 \times 10^{15} \mathrm{~W} / \mathrm{cm}^{2}$ and no auxiliary pulse. The same figure reports also a typical spectrum lineout and the temporal profile of the SRS emission compared to the laser pulse (discussed below). The emission has a complex and not reproducible spectral structure, with an overall bandwidth usually increasing with the delay between auxiliary and main pulses [Fig. 6(a)]. The intensity of this emission grows with laser energy, for a fixed delay, showing a saturation for $\Delta \mathrm{t}=1200 \mathrm{ps}$ [Fig. 6(b)].

We associate these peaks to Backward Stimulated Raman Scattering (BRS) occurring in the underdense plasma. Matching conditions and a plasma temperature of $\sim 1.5 \mathrm{keV}$, taken as an effective value during the laser peak, imply that BRS is excited in the density range of $0.10-0.15$ $n_{c}$ and drives forwardly directed EPWs with wave-vector $k_{e}=1.37-1.52 \omega_{0} / c$. The lower wavelength cutoff of the
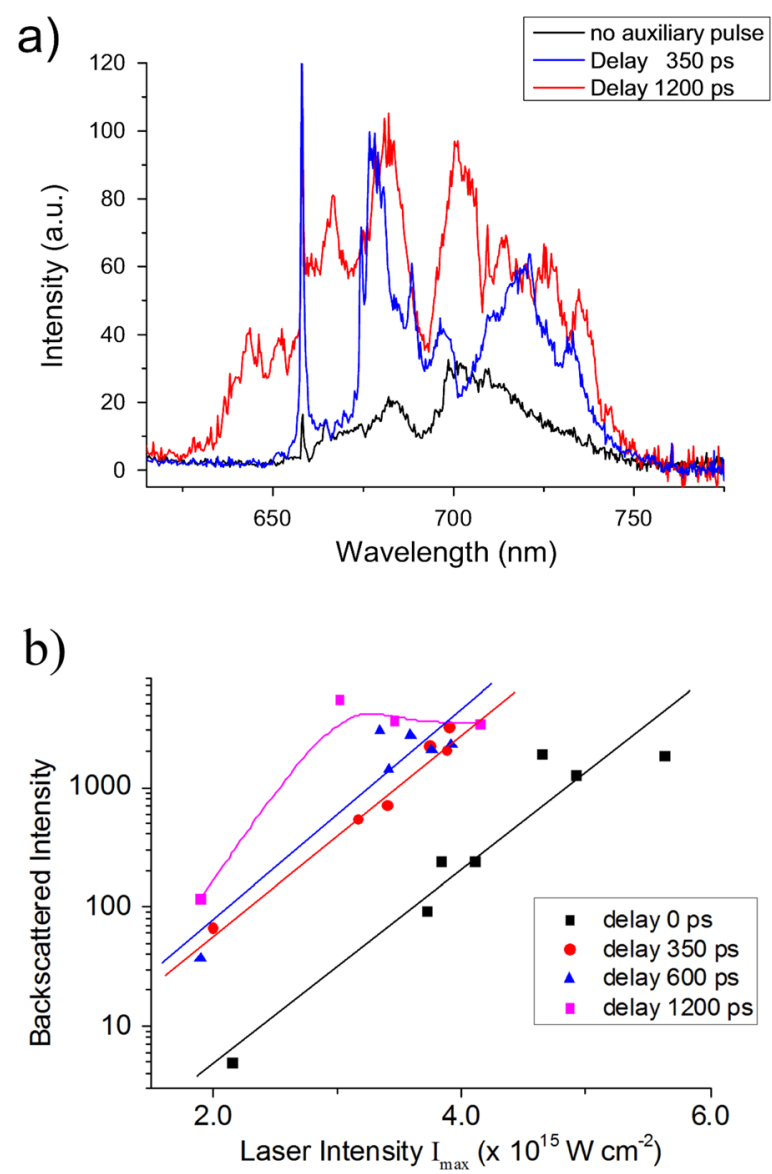

FIG. 6. (a) SRS spectra obtained with a laser intensity of $I_{\max } \approx(3.5-5.5)$ $\times 10^{15} \mathrm{~W} / \mathrm{cm}^{2}$ and delays between auxiliary and main pulses of 350 and $1200 \mathrm{ps}$, compared with the spectrum obtained without the auxiliary pulse. (b) Curves of growth of SRS intensity for different values of the auxiliarymain pulse delay. 
emission $\left(n_{e} / n_{c}=0.10\right)$ corresponds to a value of $k_{e} \lambda_{D} \approx 0.27$, which agrees with a cutoff due to Landau damping of plasma waves; this value, indirectly, confirms the value of the plasma temperature in the underdense plasma estimated above.

\section{A. SRS threshold and role of speckles}

Stimulated Raman Scattering is driven by local values of the laser intensity. An investigation of SRS threshold in the present experiment must therefore account for the distribution of e.m. fields in the micro-scale. The use of a Random Phase Plate on the laser beam results in the reduction of the longitudinal and transverse spatial coherence length, ${ }^{61}$ yielding small speckles of size $l_{\perp} \approx 2 F \lambda_{0}=1.6 \mu \mathrm{m}$ and length $l_{\|} \approx 8 F^{2} \lambda_{0} \sim 14 \mu \mathrm{m}$ ( $F$ is the $f$-number of the system). Taking into account the expected laser intensity distribution in the speckles and the number of speckles expected in the focal volume $\left(\approx 10^{5}\right)$, the maximum local laser intensity should reach $\sim 8-10 I_{\text {max }}$. Speckle dimensions and intensity distribution control both filamentation and parametric instabilities occurring during the interaction. Here, ponderomotive self-focusing of the most intense speckles with $\mathrm{I}>(2-3)$ $\times 10^{16} \mathrm{~W} / \mathrm{cm}^{2}$ is expected to occur at densities relevant for SRS and TPD processes, while for lower intensity speckles, filamentation is inhibited by their small dimensions.

The threshold of SRS instability in speckles depends on many factors, including their possible filamentation and collective effects between speckles. The issue becomes much more complex in the kinetic regime $\left(k_{e} \lambda_{D}>0.2\right)$, where the non-linear Landau damping effect, depending on the electron trapping in EPW and on their side loss across filaments, plays an important role. Here, BRS threshold is basically estimated by considering the main damping effects of electron plasma waves. In inhomogeneous density profiles, the main source of damping is usually the limited resonance region where matching conditions are satisfied. The length $l$ of this region is set by imposing $\int_{o}^{l} \kappa d x \approx 1 / 2$, where $\kappa$ $=k_{0}-k_{s}-k_{e}$ is the wavenumber mismatch of the interacting waves. Here, assuming a linear density profile in the resonant region, we calculate $l \sim 1.5 \mu \mathrm{m} \approx 3.4 \lambda_{0}$, resulting in an effective damping rate of $v_{s} / l=4 \times 10^{-2} \omega_{0}$ for the scattered e.m. wave and of $v_{e} / l=1.53 \times 10^{-3} \omega_{0}$ for the EPW, where $v_{s}$ and $v_{e}$ are the group velocities of the two waves.

Relying on classical theory, the growth of convective SRS can be expressed by $I_{S R S}=I_{\text {noise }} \exp (2 \pi \lambda)$ with the Rosenbluth parameter $\lambda=\gamma_{0}^{2} / \kappa^{\prime}\left|v_{e} v_{s}\right|=\left(\gamma_{0} l / v_{s}\right)\left(\gamma_{0} l / v_{e}\right)$, where $\gamma_{0}$ is the homogenous growth rate, and the two terms in brackets correspond to the number of e-folds in length $l$ of the scattered e.m. wave and of EPW, respectively. ${ }^{62}$ It is useful to plot the value of spectrally integrated intensity $I_{S R S}$ versus the product $I \cdot L$ where laser intensity $I$ is proportional to $\gamma_{0}^{2}$ and density scalelength $L$ is proportional to $1 / \kappa^{\prime}$ (see, e.g., Liu et $a l .{ }^{63}$ ). Given the time and space dependences of laser intensity, we here considered the intensity $I_{a v}$ averaged over one standard deviation in time and space $\left(\mathrm{I}_{\mathrm{av}}=\mathrm{I}_{\max } /\right.$ 1.366). Density scalelength values $L$ were taken from the DUED and CHIC hydrodynamic simulations for different shot conditions (intensity and delay).
We observe that this representation-shown in the plot of Fig. 7-strongly reduces the scatter of points as compared to what obtained in the graph of $I_{S R S}$ vs. $I$ [Fig. 6(b)] and therefore seems to effectively account for both the effects of laser intensity and density scalelength; besides, the approaching of points supports the hypothesis that the Rosenbluth parameter, via the product $I \cdot L$, is the parameter determining the SRS threshold and growth in our experiment. Figure 7 also shows that the BRS emission rapidly grows for smaller $I_{a v} \cdot L$ values and tends to saturate at larger values.

According to Liu et al., ${ }^{63}$ the threshold for BRS in inhomogeneous plasmas, obtained by considering wavenumber mismatch conditions, can be calculated by $v_{0}^{2} / c^{2}>1 / k_{0} L$, where $v_{0}$ is the quiver velocity of an electron in the e.m. laser field. The threshold is therefore $I^{B R S}=(4.5-8) \times 10^{15} \mathrm{~W} / \mathrm{cm}^{2}$ depending on the auxiliary-main pulse delay. It is worth noting that in Fig. 7, the threshold is represented by the red vertical line $I_{\text {thres }} \cdot L=500 \times 10^{15} \mathrm{~W} \mu \mathrm{m} \mathrm{cm}^{-2}$, showing that SRS threshold is above the laser intensity in all our shots. Such a value can be inaccurate for several reasons, among them the effect of Landau damping to be considered in the kinetic regime, the damping due to the side-loss of electrons across the speckle edge, and the possible flattening of the density profile due to filamentation of speckles, which all need 3D fully kinetic simulations to be correctly estimated. The calculated threshold, however, suggests that local intensity in speckles, higher than the envelope laser intensity, is needed to drive BRS. At the lowest laser intensities, only speckles with intensities five times higher than $I_{a v}$ can drive BRS. Conversely, at higher laser intensities, also speckles with local intensity marginally higher than $I_{a v}$ can play a role.

This picture is further supported by considering other EPW damping processes, beyond the escape of the EPW from the resonance region. Considering the present interaction conditions $\left(\mathrm{T}_{\mathrm{e}}=1.5 \mathrm{keV}, \lambda_{\mathrm{SRS}}=690 \mathrm{~nm}, n_{e} / n_{c} \approx 0.12\right.$, and speckle size, $\left.l_{\perp} \approx 1.6 \mu \mathrm{m}\right)$, Landau and side-loss ${ }^{64}$ damping rates are $\gamma_{L} \approx 5 \times 10^{-4} \omega_{0}$ and $\gamma_{S L} \approx 2 v_{t h} / l_{\perp}=1.5 \times 10^{-3} \omega_{0}$, while collisional damping $\nu_{\mathrm{ei}}$ is an order of magnitude lower. It is

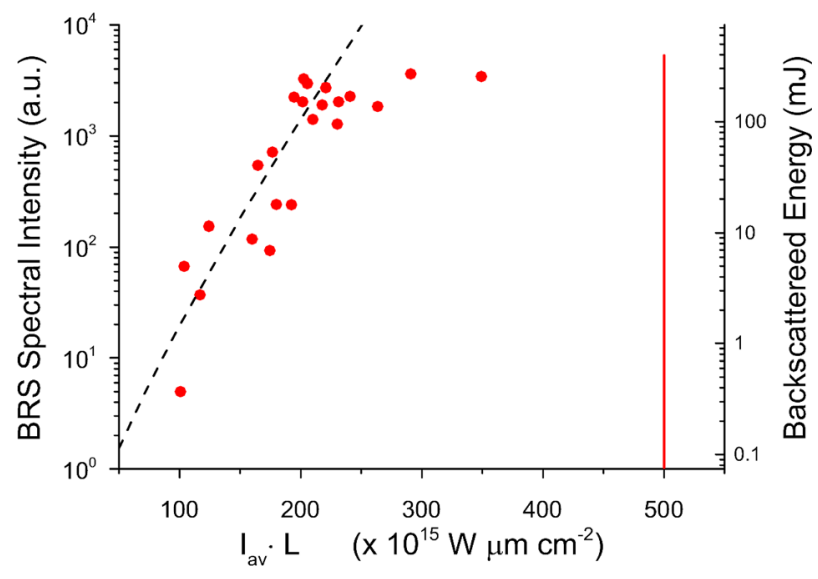

FIG. 7. BRS intensity versus the product $I_{a v} \cdot L$ appearing in the Rosenbluth parameter, where $I_{a v}$ is the laser intensity averaged over one standard deviation in time and space. The dashed line represents the rate of BRS expected from Rosenbluth theory, calculated for single pulse irradiation. 
therefore evident that side loss of electrons, due to the low $\mathrm{f} / \#$ number of focusing optics, could be more effective in limiting the convective gain than the Landau damping of EPWs, affecting the saturation of SRS. This picture however changes when the SRS is driven at $n_{e} / n_{c} \approx 0.09-0.10$, i.e., near the Landau cutoff, where the Landau damping grows and can become predominant. When taking into account both convection out of the resonance region and side loss damping of EPWs, BRS threshold shifts to values near $I_{\text {thres }} \cdot L=950 \times 10^{15} \mathrm{~W} \mu \mathrm{m} \mathrm{cm}^{-2}$ (with a slight dependence on $L$ ), which implies an even stronger role of high intensity speckles. A more accurate investigation of the effect of high intensity speckles on the BRS amplification in the present data can be found in the study by Cristoforetti et al., ${ }^{65}$ where the experimental results are compared to those obtained by the radiative-hydrodynamic code CHIC. In addition to laser refraction and diffraction, the code also accounted for the laser intensity statistics contained in the beam speckles and included self-consistent calculations of non-linear laser plasma interactions. ${ }^{30}$ The results of the simulations clearly show that a correct modeling of the speckle intensity statistics into the beam allows the experimentally observed SRS reflectivity to be explained.

\section{B. Saturation and kinetic effects}

Figures 6(b) and 7 show a rapid BRS growth and a saturation at higher intensities and scalelengths, visible, in particular, at delays of 600 and 1200 ps. SRS growth with $I_{a v} \cdot L$ is expected to be a combined effect of the larger intensity in single speckles and of the increasing number of speckles overcoming $I_{\text {thres }}$. The comparison of the experimental SRS intensities with the SRS growth predicted by the classical Rosenbluth growth rate, ${ }^{62}$ shown in Fig. 7, reveals that the convective theory well reproduces the experiment for $I_{a v} \cdot L<240 \times 10^{15} \mathrm{~W} \mu \mathrm{m} \mathrm{cm}^{-2}$, i.e., before that, the SRS saturation becomes evident. The expected SRS growth rate, displayed by the dashed line in Fig. 7, is obtained by considering single pulse irradiation and supposing that the SRS signal observed at $I_{a v} \cdot L=100 \times 10^{15} \mathrm{~W} \mu \mathrm{m} \mathrm{cm}^{-2}$ is originated by speckles immediately above the threshold. The gain here is not calculated from thermal noise but just fitting the points at lower intensities. It is worth to remark that the predicted curve does not include the fact that an increasing number of speckles contributes to BRS intensity for larger values of $I_{a v}$, which would make the curve steeper than that plotted in the graph, increasing the discrepancy with experimental results, in particular in the saturation region. The SRS saturation observed at the highest $I_{a v} \cdot L$ values suggests that damping and kinetic effects lead to saturation of SRS into the speckles.

A similar growth followed by a rapid saturation has also been found in experiments carried out at the Trident laser facility ${ }^{17}$ aimed at investigating SRS occurring in single hot spots and in PIC simulations in the kinetic regime reported in the literature. ${ }^{19,66-68}$ In these works, the saturation is due to a nonlinear frequency detuning occurring in large EPWs excited in the BRS process. ${ }^{69,70}$ The frequency shift can be due to ponderomotive and electron trapping effects as, for example, in bowing and filamentation of plasma waves in speckles. ${ }^{19,66}$ It was found ${ }^{23,67,68,71,72}$ that the nonlinear phase detuning results also in a non-stationary Raman saturation, in the form of a transition from a strongly modulated quasi-periodic to intermittent chaotic regime, with an increasing laser intensity. ${ }^{23,73}$ Since the SRS saturation is not mainly determined by Landau damping but rather by convective and side loss effects, we do not expect inflationary SRS or autoresonance ${ }^{74}$ effects due to the ponderomotive trapping of electrons to be relevant for SRS growth, as discussed by Vu et al. ${ }^{64}$

The occurrence of kinetics mechanisms in BRS saturation in the present experiment is suggested by the chaotic character of spectra in Fig. 6(a). Intensity dependent incoherent spectral broadening could be, in fact, attributed to nonlinear saturation of BRS with large bursts and quasi-periodic pulsations in intensity, as observed in kinetic simulations. $^{71,72}$ According to theory and PIC simulations, kinetic mechanisms are expected to result in non-stationary SRS saturation and reflectivity bursts of the duration of the order of the ps or lower. ${ }^{21,72,73}$ Time-resolved BRS spectra in the saturation region $\left(I_{a v} \cdot L>240 \times 10^{15} \mathrm{~W} \mu \mathrm{m} \mathrm{cm}^{-2}\right)$ with high temporal resolution $(\Delta \mathrm{t}=8 \mathrm{ps})$ show evidence of emission bursts, as shown in Fig. 8. The temporal lineout of streak images reveals that the typical time duration of emission bursts is $\mathrm{FWHM} \approx 8-10$ ps, i.e., limited by the time resolution of the streak-camera. This is consistent with numerical PIC results and confirms the presence of kinetic mechanisms in the SRS saturation regime.

\section{Timing of SRS emission}

As pointed out above, the temporal evolution of SRS reflectivity exhibits a burst-like behavior and a considerable

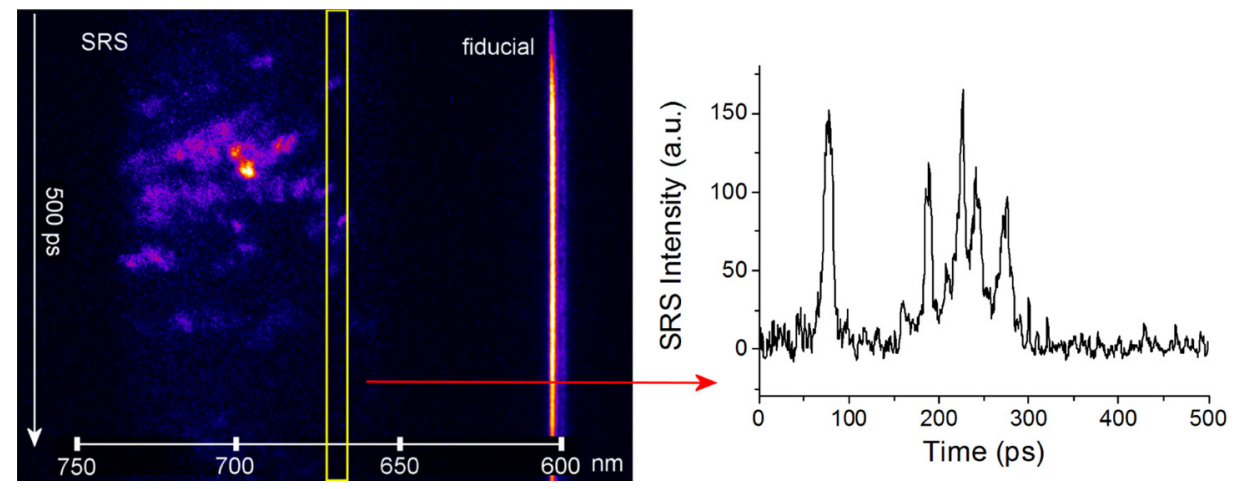

FIG. 8. (Left) Time-resolved SRS spectrum in the saturation region $\left(I_{a v} \cdot L=280 \times 10^{15} \mathrm{~W} \mu \mathrm{m} \mathrm{cm} \mathrm{cm}^{-2}\right)$ obtained with a laser intensity of $I_{\max } \approx 4.5 \times 10^{15} \mathrm{~W} / \mathrm{cm}^{2}$ and no auxiliary pulse is shown. The acquisition time window, spanning in the vertical axis, is $500 \mathrm{ps}$, and the time resolution is $\approx 8$ ps. (Right) The time profile of SRS emission in the spectral range $\lambda=670 \pm 3 \mathrm{~nm}$. 
delay with respect to the peak of the laser pulse, as shown in Fig. 5. The delay between BRS and laser peaks, measured only in the case of shots without the auxiliary pulse, was $\sim 190-220$ ps. The reflectivity due to Stimulated Raman Scattering is observed at even longer times, up to $300 \mathrm{ps}$ after the laser peak, when laser intensity is very low. In order to understand this trend, hydrodynamic simulations with the radiative-hydrodynamic code $\mathrm{CHIC}$ were carried out. As in the study by Cristoforetti et al., ${ }^{65}$ simulations included selfconsistent calculations of Stimulated Raman Scattering and generation of hot electrons by appropriate scaling laws using the local and instantaneous values of laser intensity and plasma parameters. The laser temporal profile measured shot by shot in the PALS control room was used in the simulations.

Simulation results obtained for $I_{\max } \approx 3.7 \times 10^{15} \mathrm{~W} / \mathrm{cm}^{2}$ and no auxiliary pulse are shown in Fig. 9. Here, the code was not optimized to reproduce the speckles with the highest laser intensity. For this reason, local laser intensity barely overcomes the SRS threshold in a few regions of the laser spot, which explains the low value of the hot electron flux obtained. Consistent with our experimental findings, simulations show that SRS is driven in the trailing part of the laser pulse, here at $\sim 180$ ps after the peak of the pulse. Such a value is understood by looking at the temporal growth of the density scalelength in the plasma, which progressively increases during the laser pulse and reaches the maximum value when the laser pulse is over. Moreover, as shown in Fig. 2(b), the plasma temperature significantly decreases in the trailing part of the pulse, resulting in a considerable reduction of Landau and side loss damping rates and then in a corresponding lowering of the SRS threshold. These facts explain why the optimal conditions for SRS onset occur in the tail of the laser pulse.

\section{HOT ELECTRONS}

A suprathermal electron propagating into the target gives rise to $\mathrm{Cu} K_{\alpha}$ and $\mathrm{Ti} K_{\alpha}$ emission when it crosses $\mathrm{Cu}$ and Ti tracer layers. X-ray spectroscopy of $\mathrm{Cu} K_{\alpha}$ and $\mathrm{Ti} K_{\alpha}$

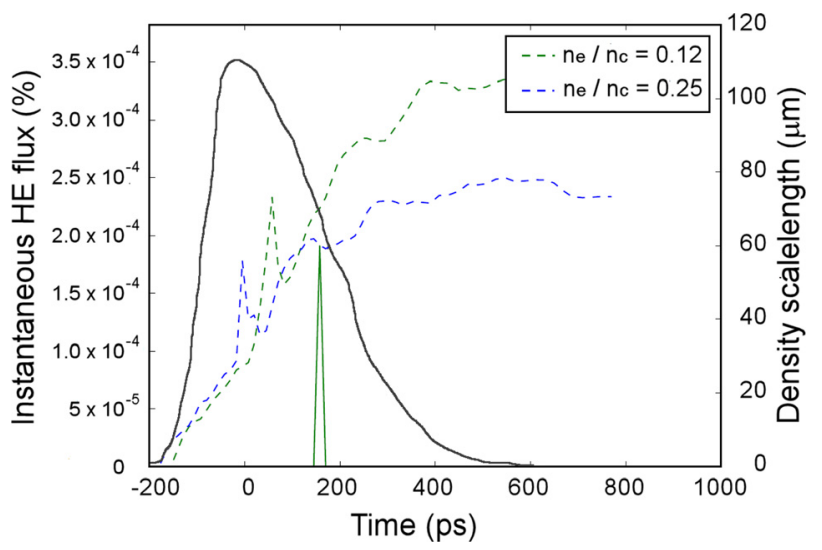

FIG. 9. Simulation results for $I_{\max } \approx 3.7 \times 10^{15} \mathrm{~W} / \mathrm{cm}^{2}$ and no auxiliary pulse: instantaneous hot electron flux driven by BRS as a function of time (green) and density scalelengths computed at $\mathrm{n}_{\mathrm{c}} / 4$ and $0.12 \mathrm{n}_{\mathrm{c}}$ (dashed blue and green lines, respectively). The intensity of the laser pulse is shown as a black line. emission is therefore here used as a diagnostics of hot electrons. The flux and average energy of HEs were here estimated by measuring the reduction of the $\mathrm{Cu} K_{\alpha}$ and $\mathrm{Ti} K_{\alpha}$ signals when the thickness of the plastic layer was increased. By considering the electron stopping range in different layers (tabulated in ESTAR database of NIST $^{75}$ ), this value allowed us to calculate the penetration depth of hot electrons and hence their average energy. Monte Carlo simulations performed with the GEANT4 ${ }^{76}$ and PENELOPE ${ }^{77}$ codes were used to reproduce the experimental results. Assuming an exponential distribution for hot electron energy $\sim \exp \left(-E / T_{\text {hot }}\right)$, a temperature $\mathrm{T}_{\text {hot }}=20 \pm 10 \mathrm{keV}$ and an energy HE conversion $\varepsilon_{H E} \sim 0.14 \% \pm 0.03 \%$ were obtained. Such distribution well fitted the $K_{\alpha}$ signal obtained for all shots with the exception of those using plastic layers of $125 \mu \mathrm{m}$ and overall $180 \mu \mathrm{m}$. In these shots, the measured $K_{\alpha}$ emission was higher than expected. This suggests that energetic HEs, with a temperature of $\mathrm{T}_{\text {hot }}>100 \mathrm{keV}$, are also generated during the interaction. Another approach for estimating hot electron energy is considering the ratio between $\mathrm{Cu} \mathrm{K}_{\alpha}$ and $\mathrm{Ti} \mathrm{K}_{\alpha}$ emission on the same shot. This value does not depend on the absolute number of generated hot electrons, which may vary shot by shot, making this diagnostic more accurate. The HE temperature obtained with this method was $\mathrm{T}_{\text {hot }}=25 \pm 5 \mathrm{keV}$, and the energy conversion was $\varepsilon_{H E} \sim 0.1 \% \pm 0.05 \%$ of the incident laser energy. Hot electron energy was also measured by using a bremsstrahlung cannon, resulting in a temperature of $T_{\text {hot }}=19 \pm 3 \mathrm{keV}$, in agreement with the previous values referring to the colder temperature.

The measured temperature of $\sim 20 \mathrm{keV}$ is close to that obtained for hot electrons generated by BRS ( $\mathrm{T}_{\text {hot }} \approx 28 \mathrm{keV}$ ) in the 1D PIC simulations of Klimo et al. ${ }^{27}$ Although the simulation time $(\approx 80 \mathrm{ps})$ is shorter than our laser pulse, the explored range of intensities $\left(2.4-24 \mathrm{PW} / \mathrm{cm}^{2}\right)$ and the preplasma scalelength $(150 \mu \mathrm{m})$ are similar to those of the present experiment. In these simulations, however, SRS occurs at densities closer to the quarter critical density, which determines the phase velocity of plasma waves induced by Raman and thus the energy of the hot electrons. Conversely, those simulations turn out in a prevailing component of hot electrons with a lower energy of $\approx 10 \mathrm{keV}$, which are accelerated in cavities seeded at quarter-critical density by SRS and TPD. In the present work, this low-energy hot electron component is not observed.

The $\mathrm{Cu} K_{\alpha}$ intensities measured using a CCD working in the single-photon regime suggest a correlation between the BRS backscattered intensity and the $K_{\alpha}$ photon number, as shown in Fig. 10. This supports a scenario in which hot electrons are mainly generated by breaking of EPW induced by BRS.

The energy of the electrons generated by BRS in the density region $0.10-0.15 n_{c}$, obtained by considering the phase velocity $v_{p h}=\omega_{e} / k_{e}$ of the driven EPW, is $\approx 17-20 \mathrm{keV}$, which is in good agreement with the measured value. This again supports the conclusion that such hot electrons, which are the main component, are produced by BRS.

Assuming that the $\omega_{0} / 2$ spectra loosely reflect the frequencies of TPD EPWs, hot electrons of different energies 


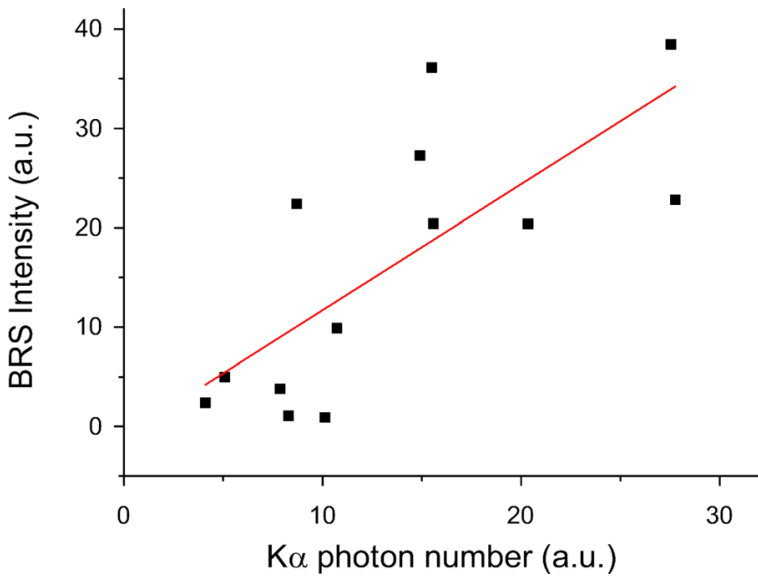

FIG. 10. Correlation between BRS intensity and $\mathrm{Cu} K_{\alpha}$ photon number measured with the CCD working in the single photon regime. Reproduced with permission from Cristoforetti et al., Europhys. Lett. 117, 35001 (2017). Copyright 2017 EPL Association. ${ }^{65}$

could be generated by the convective modes near the Landau damping and by the hybrid SRS/TPD modes near the $n_{c} / 4$ surface. EPWs in low density regions $\left(n_{e} / n_{c} \approx 0.21-0.22\right)$ are expected to easily trap thermal electrons due to their low phase velocity $\left(v_{p h} \approx 4 v_{t h}\right)$, resulting however in low-energy hot electrons $\left(\mathrm{T}_{\mathrm{e}} \approx 10 \mathrm{keV}\right)$. A Maxwellian component in the electron distribution at this low temperature was however not clearly observed in our measurements. High frequency hybrid modes, on the other hand, could in principle generate much hotter electrons $\left(\mathrm{T}_{\mathrm{e}}>100 \mathrm{keV}\right)$, but their flux is expected to be limited by the small number of thermal electrons that can be trapped at so large EPW phase velocities $\left(v_{p h} \approx 10.5 v_{t h}\right)$. By means of 2D PIC and fluid simulations, Yan et al. ${ }^{58}$ recently showed that the amount of these highenergy hot electrons can be significantly enhanced by a staged acceleration of electrons from the low density region $\left(n_{e} / n_{c} \approx 0.21-0.22\right)$, generated by convective modes, to the high density region $\left(n_{e} / n_{c} \approx 0.24-0.25\right)$ where hybrid SRS/TPD modes operate. Such high-energy electrons, which could be deleterious in SI for the possible pre-heating of the compressed fuel, could explain the $K_{\alpha}$ signal measured for the target with large plastic thickness (125 and $180 \mu \mathrm{m})$. Since the electrons generated by hybrid SRS/TPD modes are expected to be energetic and strongly peaked in the forward direction, the generated Bremsstrahlung emission is expected to be mainly emitted in the forward direction. ${ }^{78}$ This could explain why their contribution was not detected by the Bremsstrahlung spectrometer, which was looking at the irradiated side of the target.

\section{CONCLUSIONS}

The impact of parametric instabilities at laser intensities relevant for shock ignition in a planar irradiation geometry has been investigated by both calorimetry and spectroscopy. Measurements show that the main mechanisms reducing the pulse energy transfer to the plasma are Stimulated Brillouin Scattering and laser reflection with reflectivities in the lens cone of 3\%-15\% of laser energy. The energy backscattered by Stimulated Raman Scattering is lower than $1 \%$. Both
$3 / 2 \omega_{0}$ and $\omega_{0} / 2$ harmonics are measured, indicating that that a fraction of laser energy reaches the $n_{c} / 4$ surface and drives TPD and hybrid TPD/SRS instabilities.

Stimulated Raman Scattering is driven at densities compatible with classical Landau cutoff $\left(\mathrm{n}_{\mathrm{e}}=0.10-0.15 \mathrm{n}_{\mathrm{c}}\right)$ in the trailing part of the laser pulse, where the scalelength of the plasma is larger. It results in the generation of a low flux $(\sim 0.1 \%)$ of low-energy $\left(\mathrm{T}_{\text {hot }} \sim 20 \mathrm{keV}\right)$ hot electrons. Measurements also suggest the presence of a component of high energy hot electrons $\left(\mathrm{T}_{\text {hot }}>100 \mathrm{keV}\right)$, which could be possibly generated by the hybrid TPD/SRS instability. BRS is driven in the speckles generated by the RPP, where local intensity is much higher than the envelope value. The BRS gain, its spectral modulations, and the observation of reflectivity bursts suggest the occurrence of nonlinear and kinetic effects into the speckles, affecting EPW growth and resulting in the Raman saturation. BRS extent is much lower than that measured in other experiments relevant for SI, ${ }^{11,32,34,35}$ which is partly explainable by the lower plasma scalelength.

\section{ACKNOWLEDGMENTS}

We would like to thank Professor S. Hueller for the fruitful discussions and valuable suggestions on the interpretation of Stimulated Raman Scattering data. We would like to acknowledge financial support from the LASERLAB-EUROPE Access to Research Infrastructure activity within the EC's seventh Framework Program (Grant Agreement No. 284464).

We also acknowledge the support of Eurofusion Project (No. AWP17-ENR-IFE-CEA-01). We acknowledge financial contribution from the MIUR funded PRIN Project (No. 2012AY5LEL). Participation of O.R. and M.S. in this research was partly supported by the ELI project (No. CZ.02.1.01/0.0/0.0/15_008/0000162) and High Field Initiative (No. CZ.02.1.01/0.0/0.0/15_003/0000449). Participation of F.B. to this research was supported by the CNR funded Italian research Network ELI-Italy. S. Atzeni acknowledges financial support from the project Sapienza 2016 (No. 257584). The PALS staff acknowledges financial support from the Czech Ministry of Education (LM2015083 and EF16_013/0001552) and Czech Science Foundation (1705076S). We acknowledge SciTech Precision and Rutherford Appleton Laboratory Target Fabrication Group for the supplied targets. Finally, we thank the technical staff of PALS for help in running the experiments.

${ }^{1}$ R. Betti, C. D. Zhou, K. S. Anderson, L. J. Perkins, W. Theobald, and A. A. Solodov, Phys. Rev. Lett. 98, 155001 (2007).

${ }^{2}$ L. J. Perkins, R. Betti, K. N. LaFortune, and W. H. Williams, Phys. Rev. Lett. 103, 045004 (2009).

${ }^{3}$ S. Atzeni, X. Ribeyre, G. Schurtz, A. J. Schmitt, B. Canaud, R. Betti, and L. J. Perkins, Nucl. Fusion 54, 054008 (2014).

${ }^{4}$ D. Batani, S. Baton, A. Casner, S. Depierreux, M. Hohenberger, O. Klimo, M. Koenig, C. Labaune, X. Ribeyre, C. Rousseaux, G. Schurtz, W. Theobald, and V. T. Tikhonchuk, Nucl. Fusion 54, 054009 (2014).

${ }^{5}$ S. Atzeni, A. Schiavi, and A. Marocchino, Plasma Phys. Controlled Fusion 53, 035010 (2011).

${ }^{6}$ A. Marocchino, S. Atzeni, and A. Schiavi, Phis. Plasmas 21, 012701 (2014).

${ }^{7}$ X. Ribeyre, G. Schurtz, M. Lafon, S. Galera, and S. Weber, Plasma Phys. Controlled Fusion 51, 015013 (2009). 
${ }^{8}$ A. Casner, T. Caillaud, S. Darbon, A. Duval, I. Thfouin, J. P. Jadaud, J. P. LeBreton, C. Reverdin, B. Rosse, R. Rosch, N. Blanchot, B. Villette, R. Wrobel, and J. L. Miquel, High Energy Density Phys. 17, 2-11 (2015).

${ }^{9}$ E. I. Moses, R. N. Boyd, B. A. Remington, C. J. Keane, and R. Al-Ayat, Phys. Plasmas 16, 041006 (2009).

${ }^{10}$ B. Canaud, S. Laffite, V. Brandon, and M. Temporal, Laser Part. Beams 30, 183 (2012).

${ }^{11}$ W. Theobald, R. Nora, W. Seka, M. Lafon, K. S. Anderson, M. Hohenberger, F. J. Marshall, D. T. Michel, A. A. Solodov, C. Stoeckl, D. H. Edgell, B. Yaakobi, A. Casner, C. Reverdin, X. Ribeyre, A. Shvydky, A. Vallet, J. Peebles, F. N. Beg, M. S. Wei, and R. Betti, Phys. Plasmas 22, 056310 (2015)

${ }^{12}$ R. Nora, W. Theobald, R. Betti, F. J. Marshall, D. T. Michel, W. Seka, B. Yaakobi, M. Lafon, C. Stoeckl, J. Delettrez, A. A. Solodov, A. Casner, C. Reverdin, X. Ribeyre, A. Vallet, J. Peebles, F. N. Beg, and M. S. Wei, Phys. Rev. Lett. 114, 045001 (2015).

${ }^{13}$ R. Betti, W. Theobald, C. D. Zhou, K. S. Anderson, P. W. McKenty, S. Skupsky, D. Shvarts, V. N. Goncharov, J. A. Delettrez, and P. B. Radha, J. Phys.: Conf. Ser. 112, 022024 (2008).

${ }^{14}$ A. R. Bell and M. Tzoufras, Plasma Phys. Controlled Fusion 53, 045010 (2011).

${ }^{15} \mathrm{Ph}$. Nicolai, J. L. Feugeas, T. Nguyen-bui, V. Tikhonchuk, L. Antonelli, D. Batani, and Y. Maheut, Phys. Plasmas 22, 042705 (2015).

${ }^{16}$ R. K. Kirkwood, J. D. Moody, J. Kline, E. Dewald, S. Glenzer, L. Divol, P. Michel, D. Hinkel, R. Berger, E. Williams, J. Milovich, L. Yin, H. Rose, B. MacGowan, O. Landen, M. Rosen, and J. Lindl, Plasma Phys. Controlled Fusion 55, 103001 (2013).

${ }^{17}$ D. S. Montgomery, J. A. Cobble, J. C. Fernández, R. J. Focia, R. P. Johnson, N. Renard-LeGalloudec, H. A. Rose, and D. A. Russell, Phys. Plasmas 9, 2311 (2002).

${ }^{18}$ S. Depierreux, J. Fuchs, C. Labaune, A. Michard, H. A. Baldis, D. Pesme, S. Hüller, and G. Laval, Phys. Rev. Lett. 84, 2869 (2000).

${ }^{19}$ L. Yin, B. J. Albright, H. A. Rose, K. J. Bowers, B. Bergen, D. S. Montgomery, J. L. Kline, and J. C. Fernández, Phys. Plasmas 16, 113101 (2009).

${ }^{20}$ L. Yin, B. J. Albright, K. J. Bowers, W. Daughton, and H. A. Rose, Phys. Rev. Lett. 99, 265004 (2007).

${ }^{21}$ L. Yin, B. J. Albright, H. A. Rose, D. S. Montgomery, J. L. Kline, R. K. Kirkwood, P. Michel, K. J. Bowers, and B. Bergen, Phys. Plasmas 20, 012702 (2013).

${ }^{22}$ R. Yan, J. Li, and C. Ren, Phys. Plasmas 21, 062705 (2014).

${ }^{23}$ M. M. Skoric, M. S. Jovanovic, and M. R. Rajkovic, Phys. Rev. E 53, 4056 (1996); M. M. Skoric and M. M. Jovanovic, AIP Conf. Proc. 318, 380 (1994).

${ }^{24}$ H. X. Vu, D. F. DuBois, and B. Bezzerides, Phys. Plasmas 9, 1745 (2002).

${ }^{25}$ S. Depierreux, V. Yahia, C. Goyon, G. Loisel, P.-E. Masson-Laborde, N. Borisenko, A. Orekhov, O. Rosmej, T. Rienecker, and C. Labaune, Nat. Commun. 5, 4158 (2014).

${ }^{26}$ C. Riconda, S. Weber, V. T. Tikhonchuk, and A. Héron, Phys. Plasmas 18, 092701 (2011).

${ }^{27}$ O. Klimo and V. T. Tikhonchuk, Plasma Phys. Controlled Fusion 55, 095002 (2013).

${ }^{28}$ O. Klimo, J. Psikal, V. T. Tikhonchuk, and S. Weber, Plasma Phys. Controlled Fusion 56, 055010 (2014).

${ }^{29}$ A. Marocchino, M. Tzoufras, S. Atzeni, A. Schiavi, P. D. Nicolai, J. Mallet, V. Tikhonchuk, and J. L. Feugeas, Phys. Plasmas 20, 022702 (2013).

${ }^{30}$ A. Colaïtis, G. Duchateau, X. Ribeyre, Y. Maheut, G. Boutoux, L. Antonelli, Ph. Nicolai, D. Batani, and V. Tikhonchuk, Phys. Rev. E 92, 41101 (2015).

${ }^{31}$ A. Marocchino, S. Atzeni, and A. Schiavi, New J. Phys. 17, 043052 (2015).

${ }^{32}$ W. Theobald, R. Nora, M. Lafon, A. Casner, X. Ribeyre, K. S. Anderson, R. Betti, J. A. Delettrez, J. A. Frenje, V. Yu. Glebov, O. V. Gotchev, M. Hohenberger, S. X. Hu, F. J. Marshall, D. D. Meyerhofer, T. C. Sangster, G. Schurtz, W. Seka, V. A. Smalyuk, C. Stoeckl, and B. Yaakobi, Phys. Plasmas 19, 102706 (2012).

${ }^{33}$ M. Hohenberger, W. Theobald, S. X. Hu, K. S. Anderson, R. Betti, T. R. Boehly, A. Casner, D. E. Fratanduono, M. Lafon, D. D. Meyerhofer, R. Nora, X. Ribeyre, T. C. Sangster, G. Schurtz, W. Seka, C. Stoeckl, and B. Yaakobi, Phys. Plasmas 21, 022702 (2014).

${ }^{34}$ C. Goyon, S. Depierreux, V. Yahia, G. Loisel, C. Baccou, C. Courvoisier, N. G. Borisenko, A. Orekhov, O. Rosmej, and C. Labaune, Phys. Rev. Lett. 111, 235006 (2013).
${ }^{35}$ S. D. Baton, M. Koenig, E. Brambrink, H. P. Schlenvoigt, C. Rousseaux, G. Debras, S. Laffite, P. Loiseau, F. Philippe, X. Rybeyre, and G. Schurtz, Phys. Rev. Lett. 108, 195002 (2012).

${ }^{36}$ S. Depierreux, P. Loiseau, D. T. Michel, V. Tassin, C. Stenz, P. E. Masson-Laborde, C. Goyon, V. Yahia, and C. Labaune, Phys. Plasmas 19, 012705 (2012).

${ }^{37}$ J. Ullschmied, Radiat. Eff. Defect Solids 170, 278-289 (2015).

${ }^{38}$ P. Koester, L. Antonelli, S. Atzeni, J. Badziak, F. Baffigi, D. Batani, C. A. Cecchetti, T. Chodukowski, F. Consoli, G. Cristoforetti, R. De Angelis, G. Folpini, L. A. Gizzi, Z. Kalinowska, E. Krousky, M. Kucharik, L. Labate, T. Levato, R. Liska, G. Malka, Y. Maheut, A. Marocchino, P. Nicolai, T. O'Dell, P. Parys, T. Pisarczyk, P. Raczka, O. Renner, Y. J. Rhee, X. Ribeyre, M. Richetta, M. Rosinski, L. Ryc, J. Skala, A. Schiavi, G. Schurtz, M. Smid, C. Spindloe, J. Ullschmied, J. Wolowski, and A. Zaras, Plasma Phys. Controlled Fusion 55, 124045 (2013).

${ }^{39}$ D. Batani, L. Antonelli, S. Atzeni, J. Badziak, F. Baffigi, T. Chodukowski, F. Consoli, G. Cristoforetti, R. De Angelis, R. Dudzak, G. Folpini, L. Giuffrida, L. A. Gizzi, Z. Kalinowska, P. Koester, E. Krousky, M. Krus, L. Labate, T. Levato, Y. Maheut, G. Malka, D. Margarone, A. Marocchino, J. Nejdl, Ph. Nicolai, T. O'Dell, T. Pisarczyk, O. Renner, Y. J. Rhee, X. Ribeyre, M. Richetta, M. Rosinski, M. Sawicka, A. Schiavi, J. Skala, M. Smid, Ch. Spindloe, J. Ullschmied, A. Velyhan, and T. Vinci, Phys. Plasmas 21, 032710 (2014).

${ }^{40}$ T. Pisarczyk, S. Yu. Gus'kov, Z. Kalinowska, J. Badziak, D. Batani, L. Antonelli, G. Folpini, Y. Maheut, F. Baffigi, S. Borodziuk, T. Chodukowski, G. Cristoforetti, N. N. Demchenko, L. A. Gizzi, A. Kasperczuk, P. Koester, E. Krousky, L. Labate, P. Parys, M. Pfeifer, O. Renner, M. Smid, M. Rosinski, J. Skala, R. Dudzak, J. Ullschmied, and P. Pisarczyk, Phys. Plasmas 21, 012708 (2014).

${ }^{41}$ J. Badziak, L. Antonelli, F. Baffigi, D. Batani, T. Chodukowsi, G. Cristoforetti, R. Dudzak, L. A. Gizzi, G. Folpini, F. Hall, Z. Kalinowska, P. Koester, E. Krousky, M. Kucharik, L. Labate, R. Liska, G. Malka, Y. Maheut, P. Parys, M. Pfeifer, T. Pysarczyk, O. Renner, M. Rosinski, L. Ryc, J. Skala, M. Smid, C. Spindloe, J. Ullschmied, and A. ZarasSzydlowska, Laser Part. Beams 33, 561 (2015).

${ }^{42}$ L. Antonelli, P. Koester, G. Folpini, Y. Maheut, F. Baffigi, G. Cristoforetti, L. Labate, T. Levato, L. A. Gizzi, F. Consoli, R. De Angelis, Z. Kalinowska, T. Chodukowski, M. Rosinski, P. Parys, T. Pisarczyk, P. Raczka, L. Ryc, J. Badziak, J. Wolowski, M. Smid, O. Renner, E. Krousky, M. Pfeifer, J. Skala, J. Ullschmied, P. Nicolai, X. Ribeyre, G. Shurtz, S. Atzeni, A. Marocchino, A. Schiavi, C. Spindloe, T. O'Dell, Y. J. Rhee, M. Richetta, and D. Batani, J. Phys.: Conf. Ser. 688, 012003 (2016).

${ }^{43}$ L. Labate, T. Levato, M. Galimberti, A. Giulietti, D. Giulietti, M. Sanna, C. Traino, M. Lazzeri, and L. A. Gizzi, Nucl. Instrum. Methods Phys. Res., A 594, 278-282 (2008).

${ }^{44}$ C. D. Chen, J. A. King, M. H. Key, K. U. Akli, F. N. Beg, H. Chen, R. R. Freeman, A. Link, A. J. Mackinnon, A. G. MacPhee, P. K. Patel, M. Porkolab, R. B. Stephens, and L. D. Van Woerkom, Rev. Sci. Instrum. 79, 10E305 (2008).

${ }^{45}$ S. Atzeni, A. Schiavi, F. Califano, F. Cattani, F. Cornolti, D. Del Sarto, T. Liseykina, A. Macchi, and F. Pegoraro, Comput. Phys. Commun. 169, 153-159 (2005); S. Atzeni, Comput. Phys. Commun. 43, 107-124 (1986).

${ }^{46}$ J. Breil, S. Galera, and P. H. Maire, Comput. Fluids 46, 161 (2011).

${ }^{47}$ J. J. MacFarlane, I. E. Golovkin, P. Wang, P. R. Woodruff, and N. A. Pereyra, High Energy Density Phys. 3, 181 (2007)

${ }^{48}$ J. Myatt, A. V. Maximov, W. Seka, R. S. Craxton, and R. W. Short, Phys. Plasmas 11, 3394 (2004).

${ }^{49}$ W. L. Kruer, in The Physics of Laser Plasma Interactions, Frontiers in Physics, Vol. 73, edited by D. Pines (Addison-Wesley, Redwood City, CA, 1988).

${ }^{50}$ R. E. Turner, D. W. Phillion, B. F. Lasinski, and E. M. Campbell, Phys. Fluids 27, 511 (1984).

${ }^{51}$ W. Seka, B. B. Afeyan, R. Boni, L. M. Goldman, R. W. Short, K. Tanaka, and T. W. Johnston, Phys. Fluids 28, 2570 (1985).

${ }^{52}$ W. Seka, D. H. Edgell, J. F. Myatt, A. V. Maximov, R. W. Short, V. N. Goncharov, and H. A. Baldis, Phys. Plasmas 16, 052701 (2009).

${ }^{53}$ B. B. Afeyan and E. A. Williams, Phys. Rev. Lett. 75, 4218 (1995).

${ }^{54}$ R. L. Berger and L. V. Powers, Phys. Fluids 28, 2895 (1985).

${ }^{55}$ R. Yan, A. V. Maximov, and C. Ren, Phys. Plasmas 17, 052701 (2010).

${ }^{56}$ W. Seka, J. F. Myatt, R. W. Short, D. H. Froula, J. Katz, V. N. Goncharov, and I. V. Igumenshchev, Phys. Rev. Lett. 112, 145001 (2014).

${ }^{57}$ C. Z. Xiao, Z. J. Liu, C. Y. Zheng, and X. T. He, Phys. Plasmas 23, 022704 (2016) 
${ }^{58}$ R. Yan, C. Ren, J. Li, A. V. Maximov, W. B. Mori, Z.-M. Sheng, and F. S. Tsung, Phys. Rev. Lett. 108, 175002 (2012).

${ }^{59}$ D. A. Russell and D. F. DuBois, Phys. Rev. Lett. 86, 428 (2001).

${ }^{60}$ S. Weber and C. Riconda, High Power Laser Sci. Eng. 3, e6 (2015).

${ }^{61}$ D. Batani, C. Bleu, and Th. Lower, Eur. Phys. J. D 19, 231-243 (2002).

${ }^{62}$ M. N. Rosenbluth, Phys. Rev. Lett. 29, 565 (1972).

${ }^{63}$ C. S. Liu, M. N. Rosenbluth, and R. B. White, Phys. Fluids 17, 1211 (1974).

${ }^{64}$ H. X. Vu, D. F. DuBois, and B. Bezzerides, Phys. Plasmas 14, 012702 (2007).

${ }^{65}$ G. Cristoforetti, A. Colaitis, L. Antonelli, S. Atzeni, F. Baffigi, D. Batani, F. Barbato, G. Boutoux, R. Dudzak, P. Koester, E. Krousky, L. Labate, Ph. Nicolai, O. Renner, M. Skoric, V. Tikhonchuk, and L. A. Gizzi, Europhys. Lett. 117, 35001 (2017).

${ }^{66}$ L. Yin, B. J. Albright, K. J. Bowers, W. Daughton, and H. A. Rose, Phys. Plasmas 15, 013109 (2008).

${ }^{67}$ S. Miyamoto, K. Mima, M. M. Škorić, and M. S. Jovanović, J. Phys. Soc. Jpn. 67, 1281 (1998).

${ }^{68}$ C. Riconda and S. Weber, High Power Laser Sci. Eng. 4, e23 (2016).

${ }^{69}$ G. J. Morales and T. M. O'Neal, Phys. Rev. Lett. 28, 417 (1972).

${ }^{70}$ R. L. Dewar, Phys. Fluids 15, 712 (1972).
${ }^{71}$ K. Estabrook and W. Kruer, Phys. Fluids 26, 1892 (1983).

${ }^{72}$ T. Kolber, W. Rozmus, and V. T. Tikhonchuk, Phys. Fluids B 5, 138 (1993).

${ }^{73}$ M. M. Skoric, L. Nikolic, and S. Ishiguro, J. Plasma Phys. 79, 1003 (2013); M. M. Skoric, L. Nikolic, L. Hadzievski, D. Batani, S. Ishiguro, and K. Mima, J. Phys.: Conf. Ser. 688, 012112 (2016).

${ }^{74}$ T. Chapman, S. Huller, P. E. Masson-Laborde, A. Heron, D. Pesme, and W. Rozmus, Phys. Rev. Lett. 108, 145003 (2012).

${ }^{75}$ See http://physics.nist.gov/PhysRefData/Star/Text/ESTAR.html for Estar database.

${ }^{76}$ J. Sempau, E. Acosta, J. Barò, J. M. Fernandez-Varea, and F. Salvat, Nucl. Instrum. Method, B 132, 377 (1997).

${ }^{77}$ S. Agostinelli, J. Allison, K. Amako, J. Apostolakis, H. Araujo, P. Arcel, M. Asaig, D. Axen, S. Banerjee, G. Barrand et al., Nucl. Instrum. Method, A 506, 250 (2003).

${ }^{78}$ S. Von Goeler, J. Stevens, S. Bernabei, M. Bitter, T. K. Chu, P. Efthimion, N. Fisch, W. Hooke, K. Hill, J. Hosea, F. Jobes, C. Karney, J. Mervine, E. Meservey, R. Motley, P. Roney, S. Sesnic, K. Silber, and G. Taylor, Nucl. Fusion 25(11), 1515 (1985). 